\section{Military Technical College Kobry El-kobbah, Cairo, Egypt}

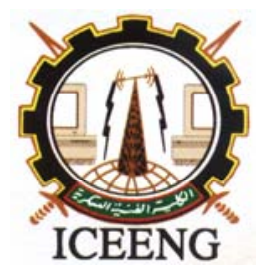

\title{
Anti-Tank Guided Missile Performance Enhancement Part-1: Hardware in the Loop Simulation
}

\author{
M.A. Abd-Altief ${ }^{1}$, G.A. El-Sheikh ${ }^{2}$ and M.Y. Dogheish ${ }^{3}$
}

\begin{abstract}
The performance of antitank guided missile systems is measured through the minimum missdistance and its capability to overcome target maneuver and different sources of errors including disturbances and noises. Toward these performance constraints, the guidance and control is considered which is one of the most interesting and challenging problem areas for antitank missile. Therefore, this paper considers an antitank guided missile system belonging to the first generation for the design and analysis. The design and analysis necessitates somehow accurate model (objective of Part-1 of the paper) for the system and a robust control design philosophy (objective of Part-2 of the paper).
\end{abstract}

Transfer functions representing the missile-control system dynamics in pitch and yaw planes are identified via hardware in the loop simulation and considered for investigation and validation against previous work and reference flight data [13]. This investigation includes experiment design, on-line identification procedure, and evaluating the identified control system (Jetvator) model within the 6DOF simulation such that the performance requirements are achieved. The results show how the hardware in the loop simulation with systemidentification lead to accurate model with clear effect upon enhancing the system performance which gave the green light for the next step of robust controller design.

Keyword: Guidance and Control, Hardware-in-Loop Simulation, System Identification, Robust Control.

\section{1- Introduction}

One of the most important command guidance systems are the antitank guided missiles (ATGM) launched against tanks and armored vehicles. These missiles are classified into three generations: first generation, second generation and third generation. In the first generation both the target and missile are manually tracked using optical telescopes. In the second generation the target is manually tracked using optical telescopes while the missile is automatically tracked by including an infrared sensor in the launcher with the telescope to detect the IR radiation from a source strapped on the rear part of the missile. Then the motion parameters are transferred automatically to signals applied to the guidance unit. Finally, the third generation is characterized by manual or automatic target tracking through optical telescopes, TV, laser or radio devices while the missile is automatically tracked by including an infrared sensor in the launcher to detect the IR radiation from a source strapped on the rear part of missile. Then the motion parameters are transferred automatically to signals applied to the guidance

\footnotetext{
${ }^{1}$ Graduate Student in Guidance and Radar Department, MTC, Cairo, Egypt

${ }^{2}$ Prof. in Electronics and Electrical Communication Department, MSA University, Cairo, Egypt

${ }^{3}$ Asc. Prof. in Electronics and Electrical Communication Department, MUST, $6{ }^{\text {th }}$ October, Cairo, Egypt
} 
unit. However, the guidance commands in this generation are transmitted to the missile through a remote link instead of wires.

Using a command link imposes some limitations upon the guidance system such as data rate of transfer, loop delay and jamming. In addition, the ever-increasing role of armored forces in modern combat directs the designers and manufacturers towards increasing the tank capabilities. These capabilities include tank power and design improvement, armor production, maneuverability of tanks and jamming. Fire efficiency has been increased by newly designed range finders-sights, stabilizing devices, and other appliances allowing the tanks to deliver effective fire during the motion and develop high combat speeds. However, the anti-tank guided missiles are usually following a parallel way for improvement and overcoming the tanks' capabilities. The performance of antitank guided missile systems is measured through the minimum miss-distance, and the capability of the missile to overcome different sources of uncertainty. One of the most interesting and challenging problem areas for antitank guided missile is that of the guidance and control. Toward this objective, a first generation antitank guided missile system is considered for the controller design and analysis.

Transfer functions representing the missile-control system dynamics in pitch and yaw planes are identified via hardware in the loop (HWIL) simulation and considered for investigation and validation against previous work and reference flight data. This investigation includes experiment design, on-line identification procedure, and evaluating the identified control system (Jetvator) model within the $6 \mathrm{DOF}$ simulation such that the performance requirements are achieved. The results show how the HWIL simulation with system-identification lead to accurate model with clear effect upon enhancing the system performance, which gave the green light for the next step of robust controller design. The robustness of the obtained model diminishes the effects of un-modeled dynamics towards enhanced system performance and gives the base for robust controller design in the next part of the paper. The evaluation is carried out for different engagement scenarios with and without inserting the hardware in the loop, where the hardware includes the jetvator drives with the electronic pack of the missile.

\subsection{Guidance and Control Loop}

The autopilot is a closed loop system and it is a minor loop inside the main guidance and control loop. Surface-to-surface missiles usually employ gyro control autopilot as shown in Fig. 1, which show a simplified block diagram of the missile guidance and control loop including the autopilot loop. The operator measures the deviation of the missile LOS direction from the target LOS direction and generates the guidance commands according to the adopted guidance law and the instantaneous missile-target positions. These guidance commands are used to control the missile motion via the autopilot. The difference between the demanded and actual acceleration is produced due to mechanical limiting in the jetvator assembly to prevent excessive acceleration demands beyond the missile structural ability. In addition, it prevents the missile from taking larger angle of attack that will cause instability or stalling. The magnitude of acceleration that the missile can perform is determined by its ability to stay in one flight condition despite the aerodynamic loading. The autopilot contains a pneumatic jetvator assemble, two gyros for pitch and way channels, and the electronic circuits.

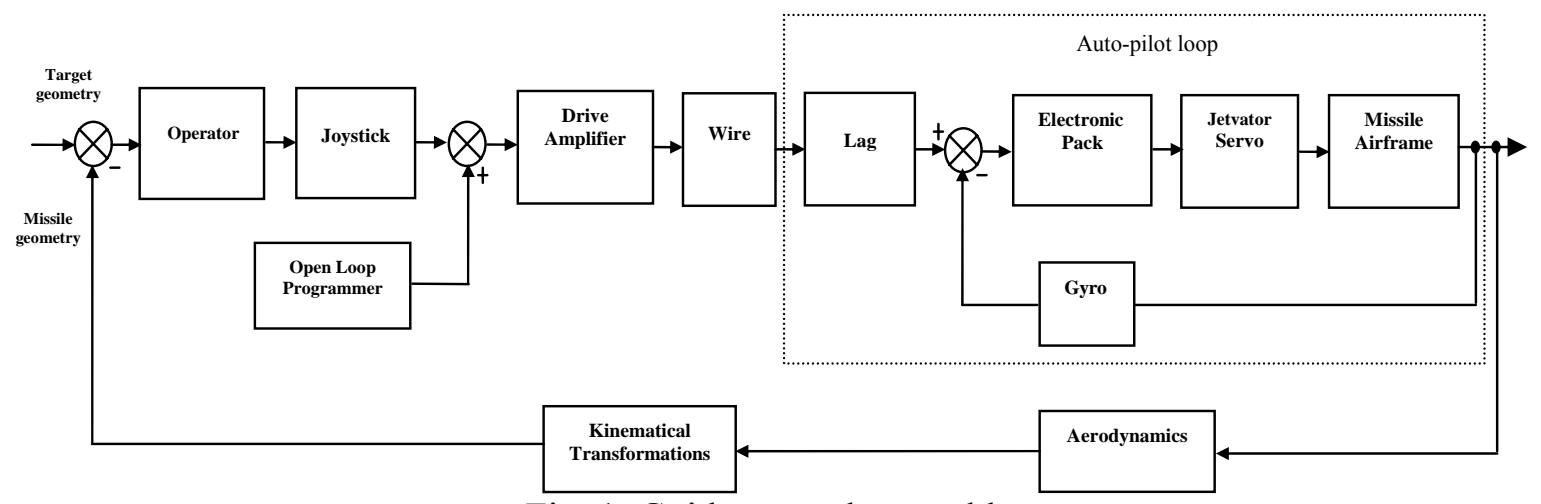

Fig. 1: Guidance and control loop 


\subsection{Electronic Package (E-Pack)}

It is the electronic circuit, which drives the actuator servos and represents the autopilot controller of the underlying system. It has two gains; low gain applied at the booster time and the high gain at the sustain time, Fig. 2. The command currents through the wire in both yaw and pitch planes are the electronic pack inputs while the output is a pulse width modulated (PWM) voltage used to drive a pair of solenoids in each plane. The input current at each channel is converted to the corresponding voltage with range of $\pm 5 \mathrm{~V}$ through lag circuit. This voltage is subtracted from the missile position voltage which picked up from gyros. The total voltage is chopped by $5 \mathrm{KHz}$ chopper with 90 degree shift in yaw channel. Both signals are multiplied by low gain at rocket engine boost time and high gain in sustain time. The gain is switched from low to high by a pressure switch mounted on the missile body. Then the pitch and yaw signals are summed and filtered by a $5 \mathrm{KHz}$ filter. The filtered signal carries both pitch and yaw command signal information.

The resolver output is $5 \mathrm{KHz}$ sinusoidal wave shifted according to the value of roll angle. That is, the resolver function is to pick up this information according to the roll angle. Then, a zero detection demodulating circuit pick the value of the filtered signal when the resolver voltage crosses the zero value and yield the output signal that represents the demodulated signal of yaw channel. The pitch demodulated signal is produced by applying zero detection demodulation sequence to 90 degree shifting of filtered signal. The previous sequence is used in order to shift the command value from pitch to yaw channels and verses while the missile flying according to the value of the roll angle.

\section{2- Hardware Implementation}

The system model should predict the input-output response in such a way that can be used to design a control system with high confidence level to work with the real physical system. The system model can be obtained either by applying the laws of physics or by conducting experiments on the physical system itself. The second approach is the hardware in the loop (HWIL) simulation in which the control section is disassembled from a real missile and interfaced with the computer simulation program via data acquisition module, Fig. 3.

The autopilot disassembled hardware section consists of actuator and jetvator assembly, Electronic Pack and resolver locked at normal position. The actuator and jetvator assembly and Electronic Pack are attached together via two potentiometers to feed back the nozzle movement. The input signal to the hardware assembly is the subtraction of the command current (through the wire link) and the gyro feedback signal. The output of the hardware is the nozzle movement voltage, which picked up through the feedback potentiometer. This voltage is transformed into jet deflection angle inside the simulation program. The input signal to the hardware section and the potentiometer voltage are transmitted from the simulation program to the hardware section and vice versa through a multi-channel input-output data acquisition module. In addition, the hardware assembly is supplied by an external power supply to feed electronic pack and a source of pressed air to feed the actuator servo.

The electronic pack has two gains; low gain for booster thrust and high gain for sustainer. They are physically controlled through pressure switch mounted on missile body and works during the missile flight. Thus, for the simulation, the pressure switch is replaced by an electric relay energized from the simulation program according to thrust value by a pulse via the data acquisition output channel. The input and output of the hardware section are recorded during the simulation run at each sample of time along the missile flight. 


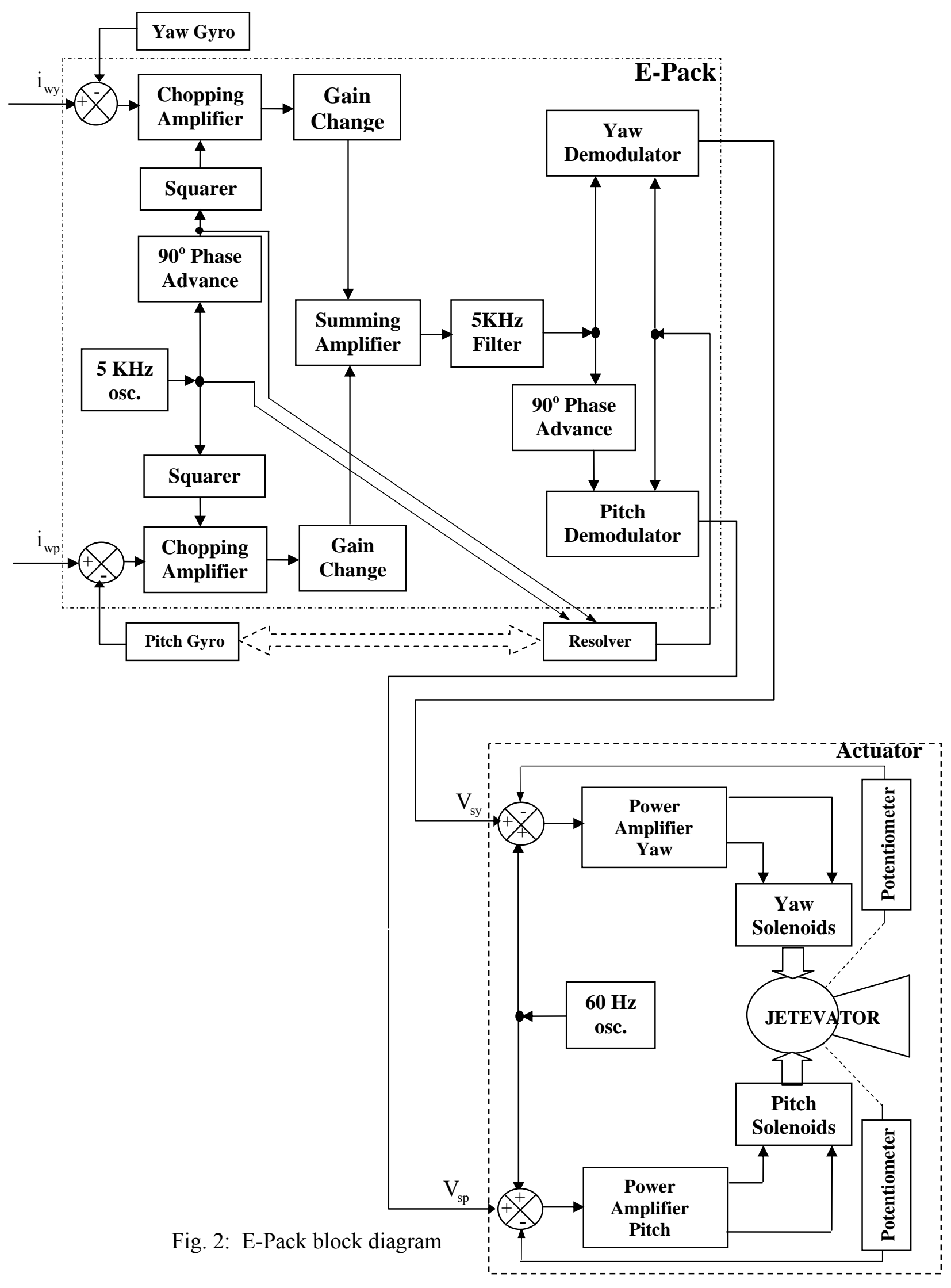




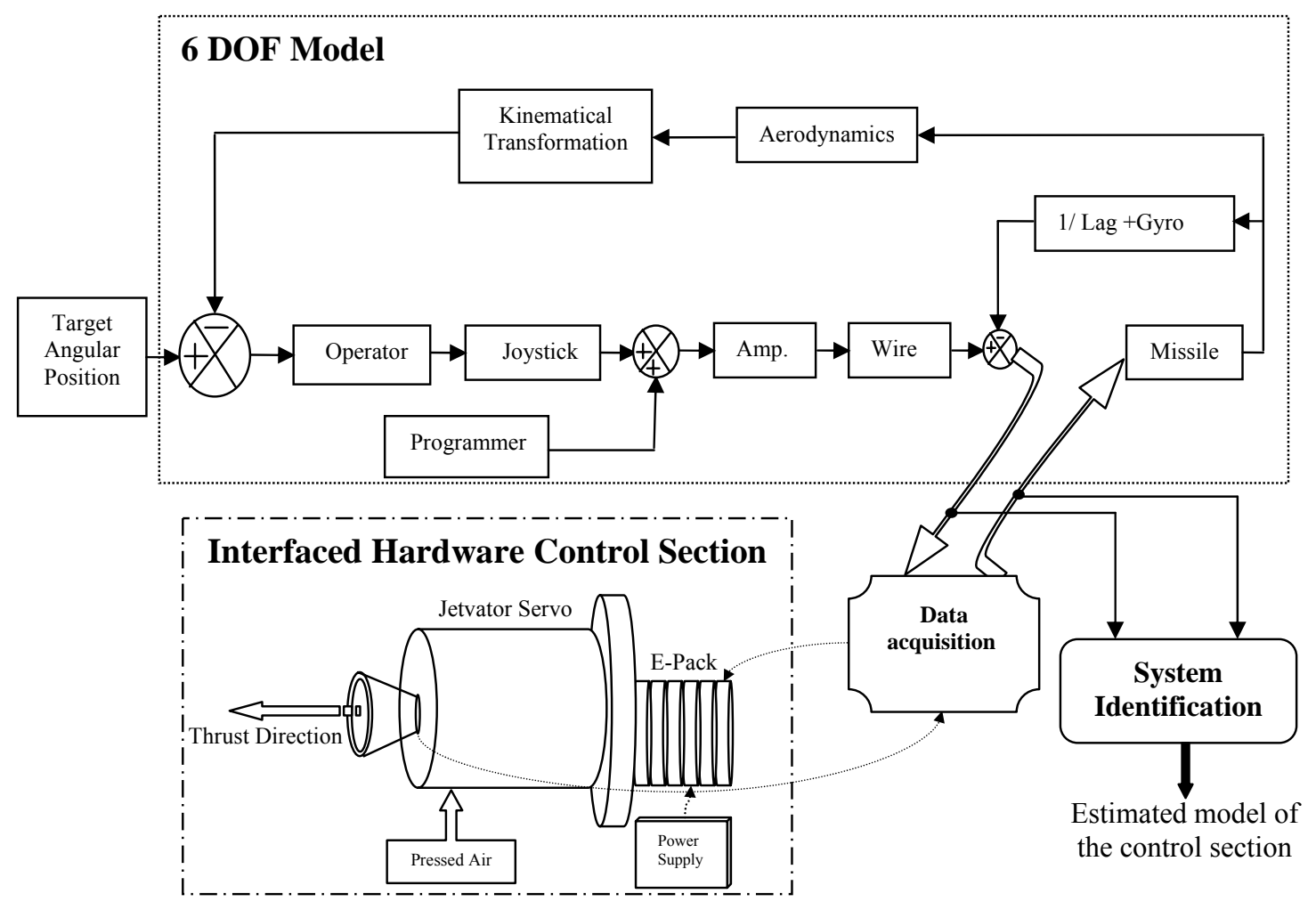

Fig. 3: Hardware in the loop simulation

Figure 4 illustrates a view for the real hardware section after disassembled from the missile while Fig. 5 shows the construction of the hardware interface experiment including the control section, computer and data acquisition module, power supply, pressed air source, and level gain switch.

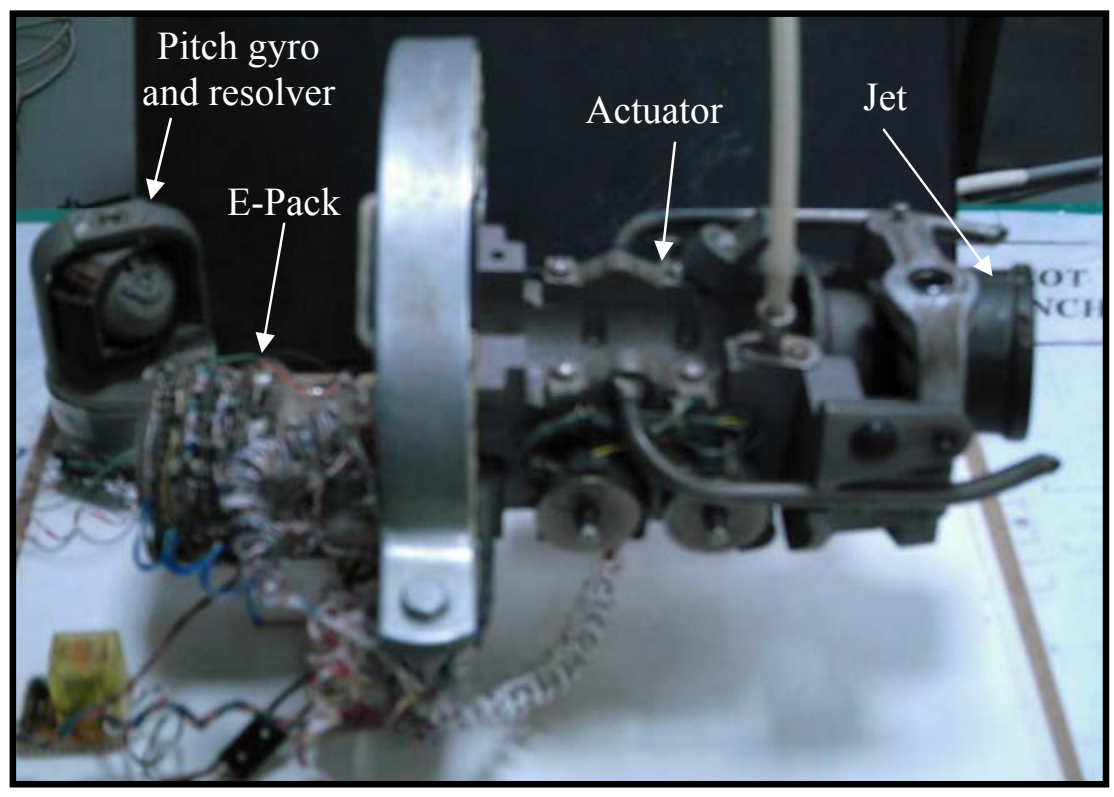

Fig. 4: Hardware control section 


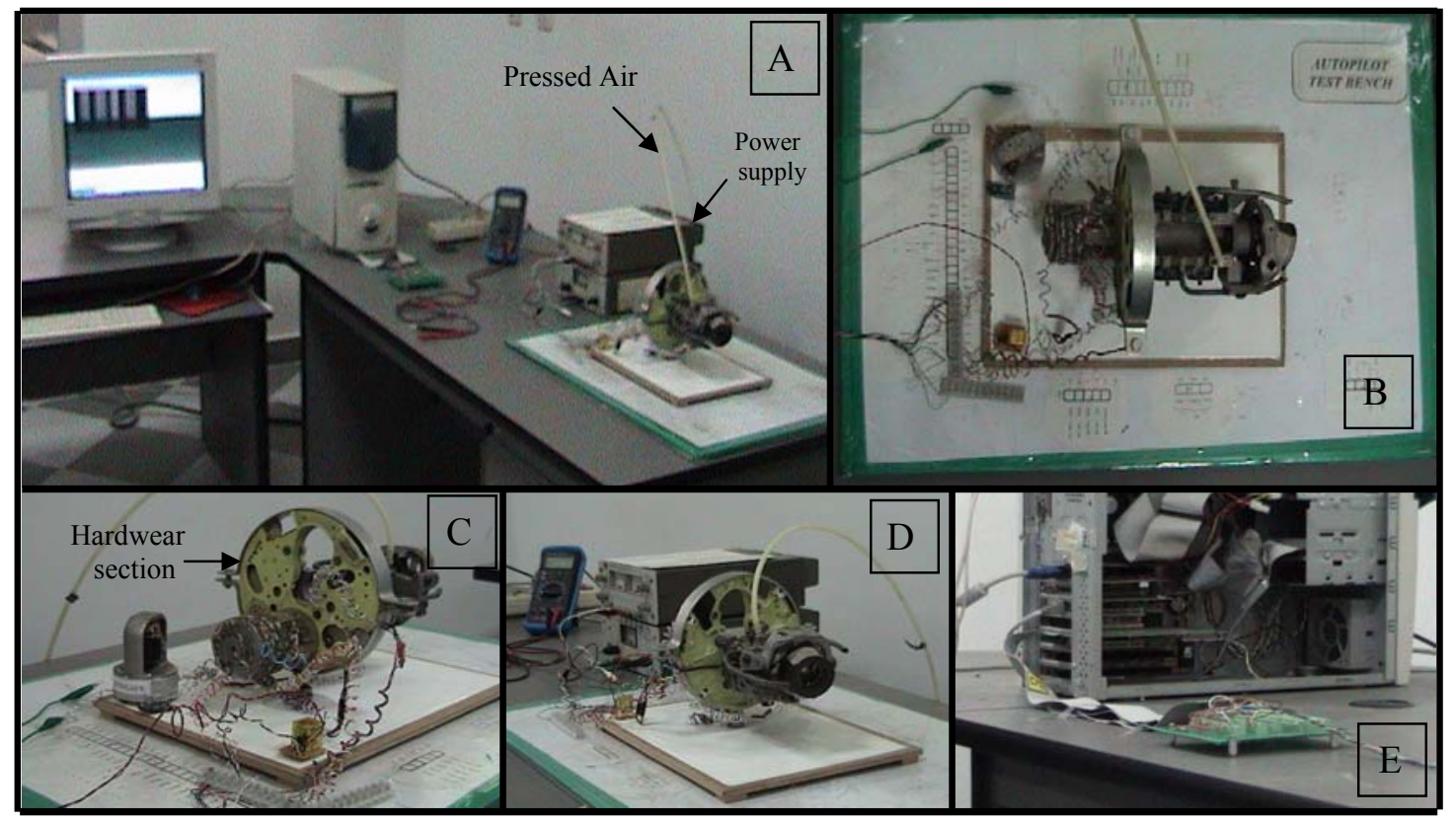

Fig. 5: Hardware in the loop experiment

\subsection{HWIL Experimental Results}

The control section simulation part is removed from the 6DOF model and replaced by the experiment connecting the hardware control section within the simulation loop. The interior signals and waveforms resulting from both the HWIL experiments and pure simulation for the control system are analyzed in addition to the flight path trajectories for both cases and reference trajectory, Fig. 6 (other figures are omitted for space). These results are obtained for target at range $\left(R_{t x}\right)=2800[\mathrm{~m}]$, velocity $\left(V_{t}\right)=0[\mathrm{~m} / \mathrm{sec}]$, pitch LOS angel $\left(\Psi_{s}\right)=0[\mathrm{mils}]$, and yaw LOS angel $\left(\theta_{s}\right)=0.25[$ mils].

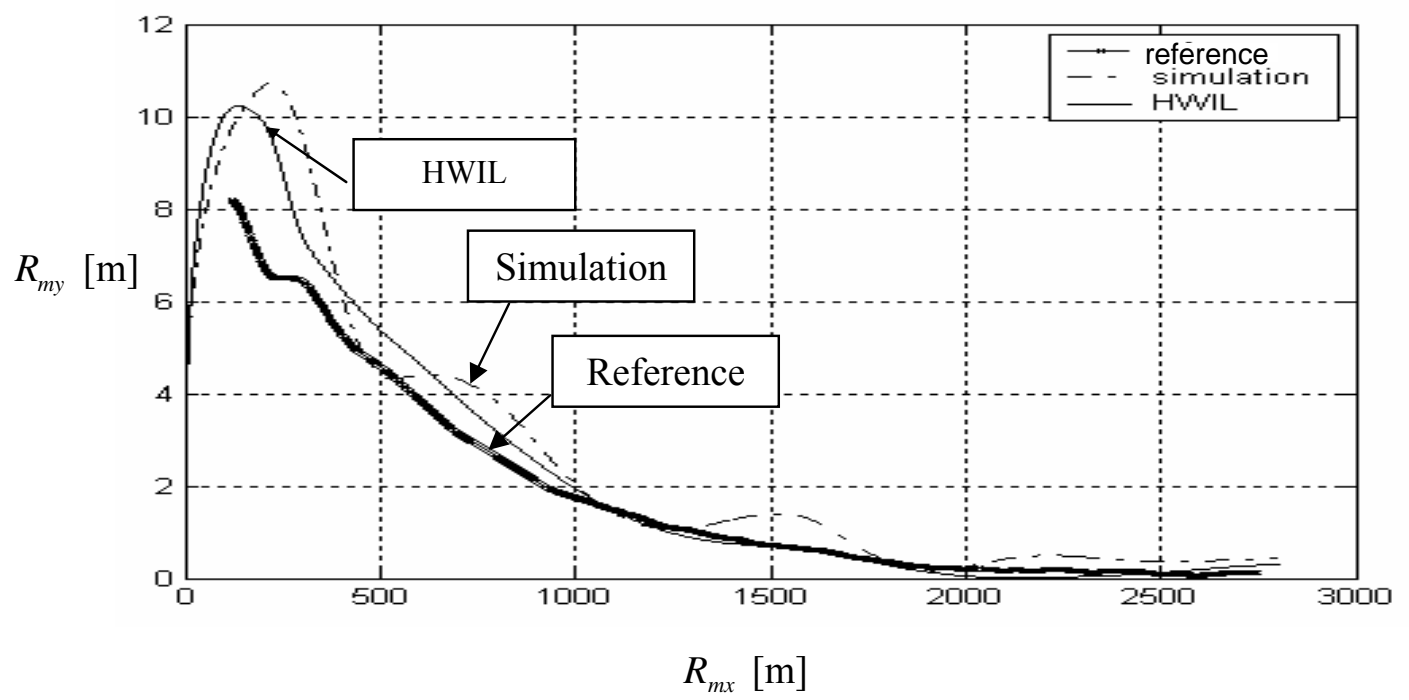

Fig. 6: Missile pitch plane trajectories reference, 6DOF model, and HWIL 
The reasons for the difference between simulation and HWIL results are due to the $60 \mathrm{~Hz}$ desire oscillation in the jetvator movement, the noise acting in case of HWIL and unmodelled dynamics in control section model. But in case of missile guidance system, it's necessary to have a 6 DOF model much close to the real system. The flight path trajectories shown in Fig. 6 clarify the smoothness of HWIL and its closeness to the reference trajectory. In addition, the difference between the $6 \mathrm{DOF}$ and the reference can be contributed to different sources of uncertainty in modeling especially the un-modeled dynamics.

\subsection{System identification}

System identification is the experimental approach to process modeling where it is used to estimate a model for the underlying plant based on measured input-output data. This procedure of identifying system parameters includes the following steps $[11,12$, 16]:

- Experiment design: its purpose is to obtain good experimental data, and it includes the choice of the input and the measured output signals.

- Selection of model structure: a suitable model structure is chosen using prior knowledge and tradeoff approach.

- Choice of the fit criterion: a suitable cost function is chosen, which reflects how well the model

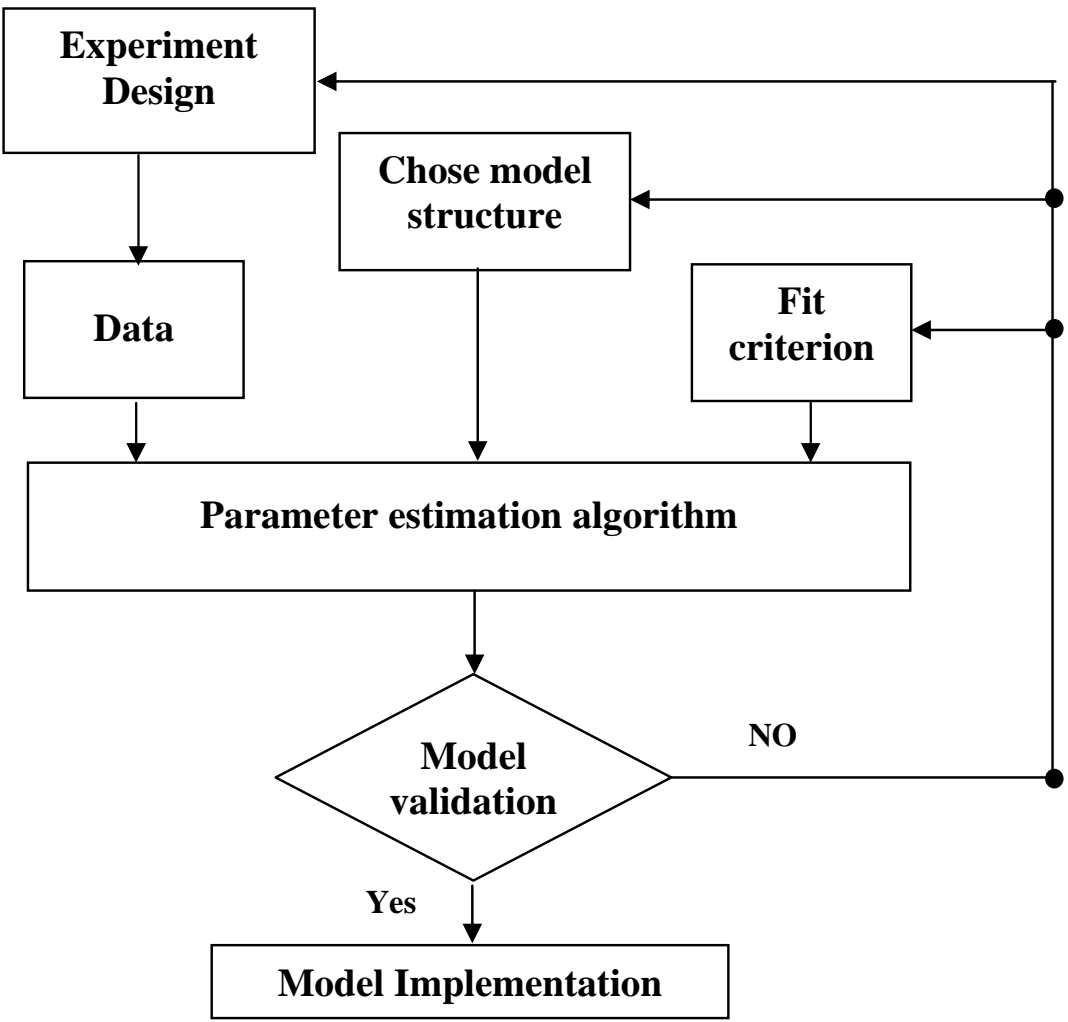

Fig. 7: System Identification procedure fits the experimental data.

- Parameter estimation: the optimization problem is solved to obtain the numerical values of the model parameters in the form of poles and zeros or the state space matrices.

- Model validation: the model is tested in order to reveal any inadequacies.

The system identification is used for building accurate and simplified models of complex systems from noisy time-series data. That is, it provides mathematical models of dynamic systems based on observed input/output data. The obtained model is validated via comparing its output with the original or observed data. Estimating the system models might be carried out using either parametric or nonparametric methods $[11,16]$. Nonparametric methods are direct estimation of the impulse or the frequency response of the system. This method does 
not impose any structure assumptions about the system, other than that it is linear. However, the parametric method is utilized with this work to find the model (state space, ARX and ARMAX) appropriate for controller design. Parametric methods necessitate a specific model structure in which the parameters are estimated using the observed input/output data. Dominating ways are state-space and several variants of difference equation descriptions such as $[1,11,12,16]$ ARX, ARMAX, Output-Error (OE) and Box-Jenkins (BJ) models. The prediction error method (PEM) $[4,17,19]$ is tailored with the underlying system.

\subsection{System Identification Algorithm}

The identification process amounts to repeatedly selecting a model structure, computing the best model in the structure, and evaluating this model's properties to justify its satisfaction. This cycle can be itemized as follows:

Step1:Design an experiment and collect input-output data from the process or system to be identified.

Step2:Examine the data, polish it so as to remove trends and outliers, and select useful portions of the original data. Possibly apply filtering to enhance important frequency ranges.

Step3:Select and define a model structure (a set of candidate system descriptions) within which a model is to be found.

Step4:Compute the best model in the selected model structure according to the input-output data and the given criterion of fit.

Step5:Examine the obtained model's properties.

Step6:If the model is good enough, then stop; otherwise go back to (Step 3) to try another model set. Possibly also try other estimation methods (Step 4) or work further on the input-output data (Steps 1 and 2).

A MATLAB program is designed and built to create transfer function models for the implemented hardware using experimental data gained from the flight simulation program. This program conducts the system identification steps and computes the best model in state space and then in transfer function forms.

\section{3- Performance Analysis}

A PWM signal is produced by triangle $60 \mathrm{~Hz}$ signal at the gate of the power amplifier to drive each pair of the solenoids in each channel. The amplifier opens when the triangle signal crosses certain level (threshold level), while at zero command the opening and closing duration are equal. When the command is received, the modulation voltage shifts the triangle signal upward or downward the amplifier opening level, so the amplifier working period change according to the value and direction of the command signal.

The transfer function which gives the relationship between the error command current $\left(i_{e p}\right)$ as input to E-Pack and the output voltage $\left(V_{s p}\right)$ from E-Pack in pitch plane, is driven from the implementation of the hardware control section inside the simulation program. The inputoutput data are used in the identification program to find out the final transfer function for the E-Pack within duration of flight time. Since the E-Pack has two modes of operation low gain at booster and high gain at sustainer, there are two transfer functions describing the E-Pack. The first one from lunching instant up to about 5 [sec] at the end of booster, while the second for the rest of the missile flight. The identification procedure is applied to the recorded data for flight time $t=0: 5$ [sec] to yield the fitted transfer function for the missile electronic 
package at booster time. Also the same procedure is applied to the rest of data for estimating the transfer function of the missile electronic package at the sustain time.

The transfer function of the missile electronic package along its flight is of second order in the form of Equation (1) and its parameters are illustrated in the Table (1).

$\frac{V_{s p}}{i_{e p}}=\frac{a_{1} s+a_{0}}{s^{2}+b_{1} s+b_{0}}$

Table (1) Electronic Pack transfer function coefficient

\begin{tabular}{|l|l|l|l|l|l|}
\hline $\begin{array}{l}\text { Duration } \\
\text { [Sec.] }\end{array}$ & Identification type & $a_{1}$ & $a_{0}$ & $b_{1}$ & $b_{0}$ \\
\hline \multirow{3}{*}{$0.0-5.0$} & State-space & $q \wedge, 7) r_{-}$ & -729.8312 & 519.67 & 1708.534 \\
\cline { 2 - 6 } & ARX & -33300 & -2968090 & 5425 & 8187253.8 \\
\cline { 2 - 6 } & ARMAX & -7835.55 & -1205214 & 1113.31 & 3317938.6 \\
\hline \multirow{3}{*}{$5.0-15.0$} & State-space & -154.704 & -1311.32 & 1153.25 & 6120.3745 \\
\cline { 2 - 6 } & ARX & 1 r,$r 4$ & -503799.8 & 3472.77 & 2984885 \\
\cline { 2 - 6 } & ARMAX & -19.5213 & -105.5947 & 140.0187 & 567.7883 \\
\hline
\end{tabular}

The step response, and bode diagram for the E-Pack state-space identified model are obtained and showed its characteristics.

\subsection{Actuator Assembly}

Jetvator servo mechanism is an electro-pneumatic servo valve and plays the role of control within the missile that necessitates fast speed response, high power output and working fidelity. It has the function of moving the thrust nozzle according to the command profile. The construction of the jetvator is given in section one with the detailed main components shown in Fig. 8. When one solenoid is energised, its ball is held against the pressure of the gas supply to close its inlet and opens one side of the piston to atmosphere. At the same time, the remaining solenoid is de-energised allowing the gas to pass through the other side of the piston. The solenoid valves are energised through drive amplifiers in the electronic pack. Under no-demand conditions each pair of ball valves is operated alternately at a frequency of $60 \mathrm{~Hz}$ with a 1:1 duty factor. This arrangement overcomes static friction and ensures rapid response. When a demand is received the duty factor is increased on one valve and decreased on the other, causing a greater mean pressure on one side of the piston that moves it with the jetvator nozzle in the required direction.

The jetvator transfer function is estimated using the identification program and the measured data from implementation of the hardware control section inside the simulation program. In this experiment, the E-Pack voltage is the input while the nozzle deflection is the output. The transfer function of the missile actuator along its flight is of second order in the form of Equation (2) and its parameters are illustrated in the Table (2).

$$
\frac{V_{s p}}{i_{e p}}=\frac{c_{1} s+c_{0}}{s^{2}+d_{1} s+d_{0}}
$$

Table (2) Actuator transfer function coefficient

\begin{tabular}{|l|l|l|l|l|l|}
\hline Duration [Sec.] & Identification type & $C_{1}$ & $C_{0}$ & $d_{1}$ & $d_{0}$ \\
\hline
\end{tabular}




\begin{tabular}{|l|l|l|l|l|l|}
\hline \multirow{3}{*}{$0.0-15.0$} & State-space & -372.454 & -159047.2 & 741.24 & 25199.8 \\
\cline { 2 - 6 } & ARX & 2130.215 & -8588428 & 4256.32 & 1731191.61 \\
\cline { 2 - 6 } & ARMAX & -366.266 & -124007.6 & 709.7 & 19526.9 \\
\hline
\end{tabular}

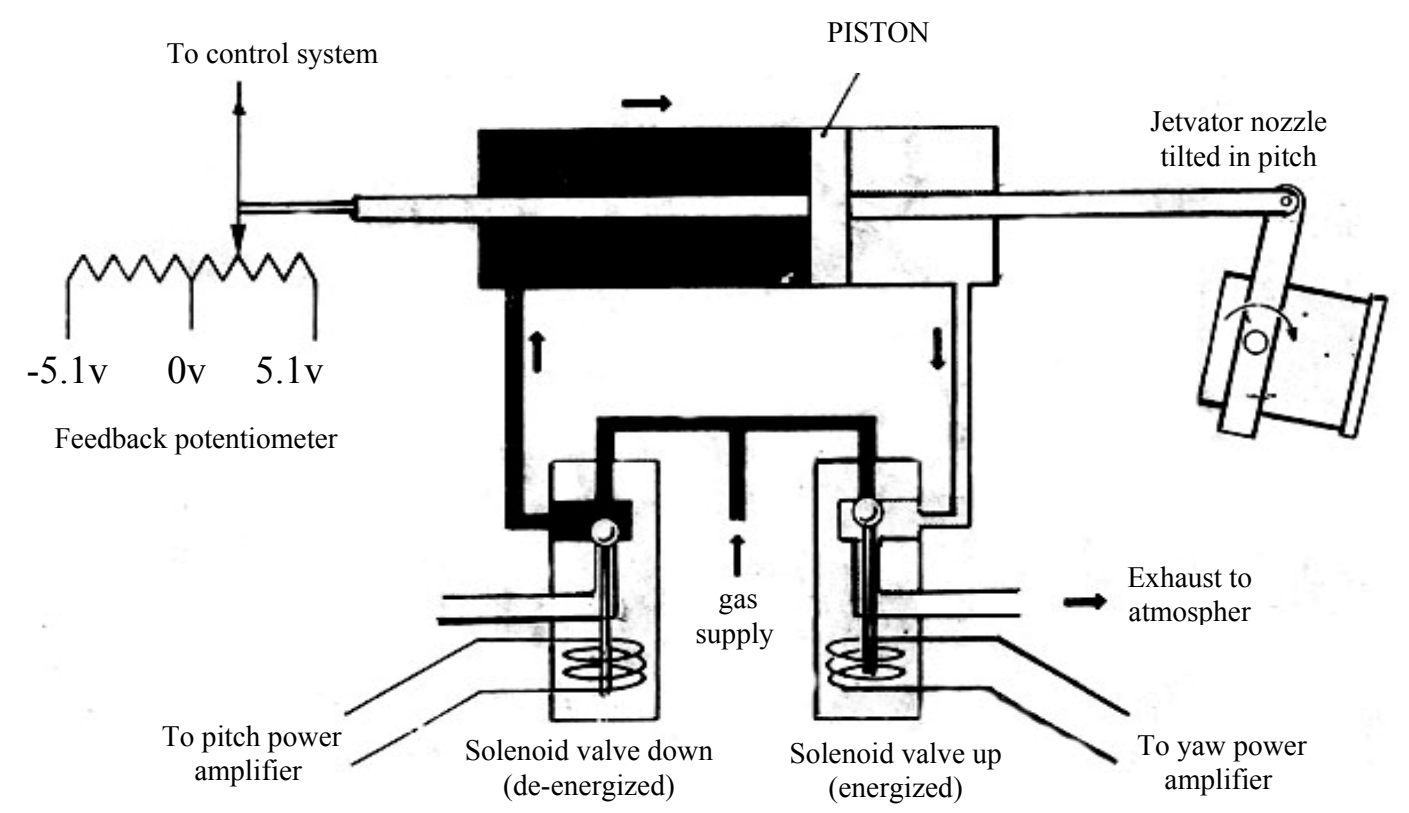

Fig. 8: Actuator function diagram

The step response and bode diagram for the state-space identified model of the actuator are shown in Fig. 9.

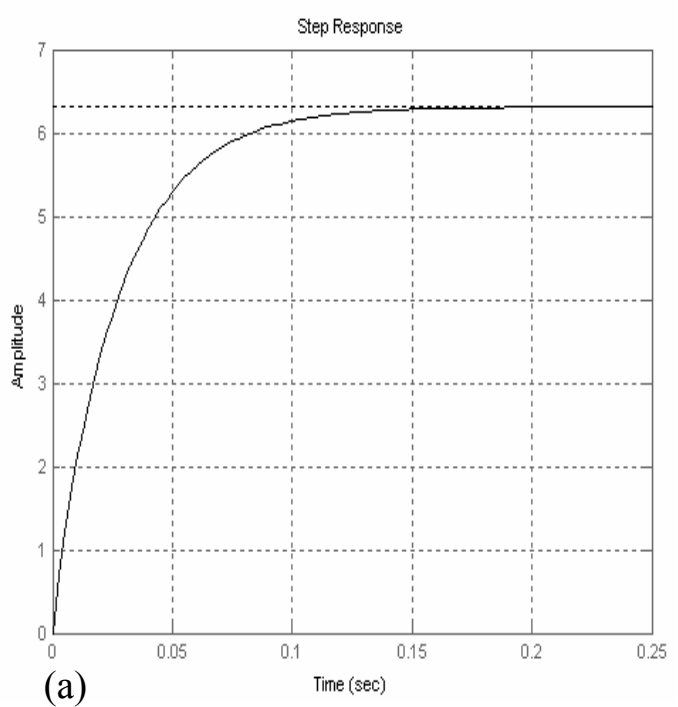

(a)

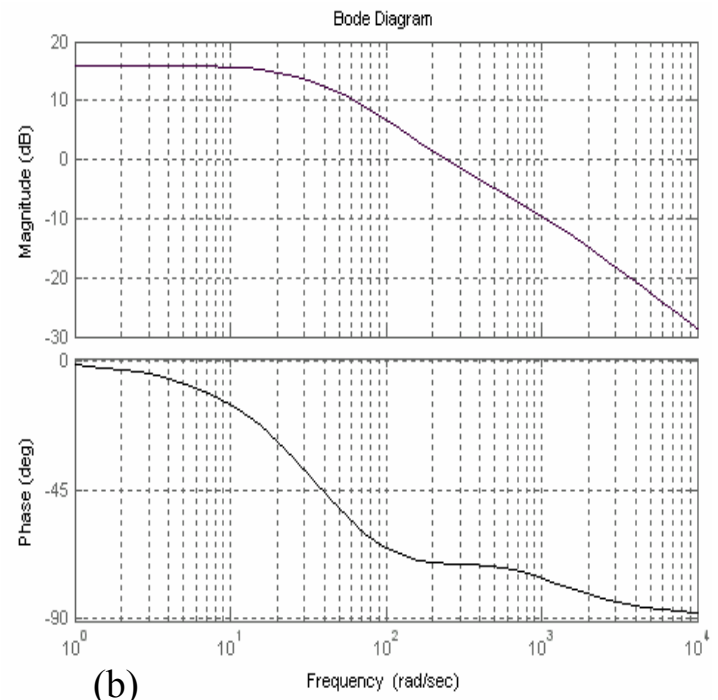

(b)

Fig. 9: Actuator state-space identified model (a) Step response and (b) bode diagram

\subsection{Missile Airframe}

Accurate determination of the missile airframe response as an element within the guidance loop constitutes a corner stone in the design of a reliable and robust guidance system. In addition, it plays an important role in the execution of the guidance commands produced by 
the guidance system. Thus, from the control point of view, the input to the missile airframe is the jet deflection for thrust vector control and its output is the missile body angels. For example, in case of pitch plane the input is the jet deflection in pitch plane $\left(\delta_{p}\right)$ and the output is the body angle in elevation $(\theta)$.

Since the missile system is time varying, there are more than one transfer function which describe the missile airframe along the flight envelope. Thus, for the purpose of analysis, the pitch data obtained form the 6DOF with HWIL are considered while the yaw channel is nearly similar. The simulation program runs using different engagements scenarios for both the HWIL and autopilot simulation model. The pitch jet deflection and corresponding body angle are recorded for a target at distance $X_{t}=2800 \mathrm{~m}$, and LOS angels in both yaw and pitch planes $\Psi_{s}=0^{\circ}$ and $\theta_{s}=1^{\circ}$. The transfer functions parameters of the airframe are identified according to the input-output data at specified flight conditions. A comparison is carried out between missile body angle obtained from different models for same jet deflection input at different flight time durations which covers the flight envelope and coincident with the missile flight phases. Then, the transfer functions of airframe-identified models can be described by a second order in the form of Equation (3) and its parameters are illustrated in the Table (3).

$$
\frac{\theta}{\delta_{p}}=\frac{e_{1} s+e_{0}}{s^{2}+f_{1} s+f_{0}}
$$

Table (3) Airframe transfer function coefficient

\begin{tabular}{|c|c|c|c|c|c|}
\hline Duration [Sec.] & Identification type & $e_{1}$ & $e_{0}$ & $f_{1}$ & $f_{0}$ \\
\hline $0.0-1.5$ & \multirow{3}{*}{ State-space } & -0.081 & -150.18 & 6.01 & 1.3334 \\
\hline $1.5-5.0$ & & -0.03189 & $\begin{array}{l}-16.199 \\
\end{array}$ & 50.0835 & 13.9536 \\
\hline $5.0-15.0$ & & -0.027142 & -28.4218 & 542.3309 & 31.6531 \\
\hline $0.0-1.5$ & \multirow{3}{*}{ ARX } & -0.07746 & -146.52 & 5.8875 & 1.5783 \\
\hline $1.5-5.0$ & & $-\cdot, \cdot 0 Y T$ & -15.7198 & 34.2052 & 10.8946 \\
\hline $5.0-15.0$ & & -0.0329 & -10.149 & 161.86 & 11.070 \\
\hline $0.0-1.5$ & \multirow{3}{*}{ ARMAX } & -0.0818 & -152.378 & 6.0482 & 1.0449 \\
\hline $1.5-5.0$ & & -0.03519 & -19.2378 & 51.0123 & 15.9043 \\
\hline $5.0-15.0$ & & -0.027545 & -23.2626 & 414.13 & 25.398 \\
\hline
\end{tabular}

The bode diagram for the state-space identified model of the airframe in the three different flight phases shown in Fig. 10. In addition, the step response for different

identified models of the airframe in the three different flight phases can be depicted.

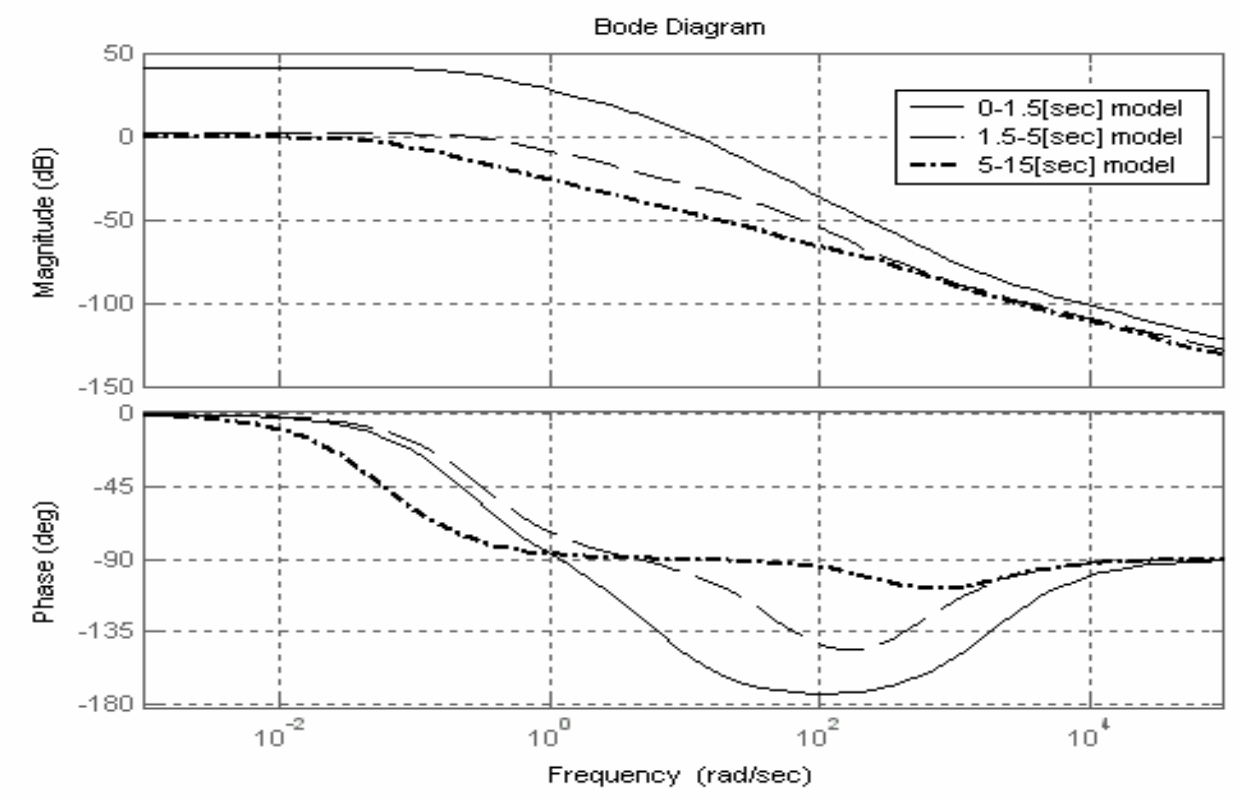

Fig. 10: Bode diagram for the airframe state-space identified model in the three different phases 


\subsection{Control System Response}

The above discussions clarify that the pneumatic autopilot has wide range of response which is varying according to the flight parameters and the operating conditions. So the simulation model should be represented for the main phases of the missile flight. The nominal simulation model for the autopilot at each phase can be selected from the previously identified models depending on the closeness to HWIL data. It was clear that the state-space models for all subsystems of the autopilot are closed to HWIL experimental data and reliable to be used for controller design. The time and the frequency responses of the autopilot with the conventional controller used with the 6DOF and the HWIL models are shown in Figures 11, 12, 13 for the different missile flight phases; running up, gathering and guidance.
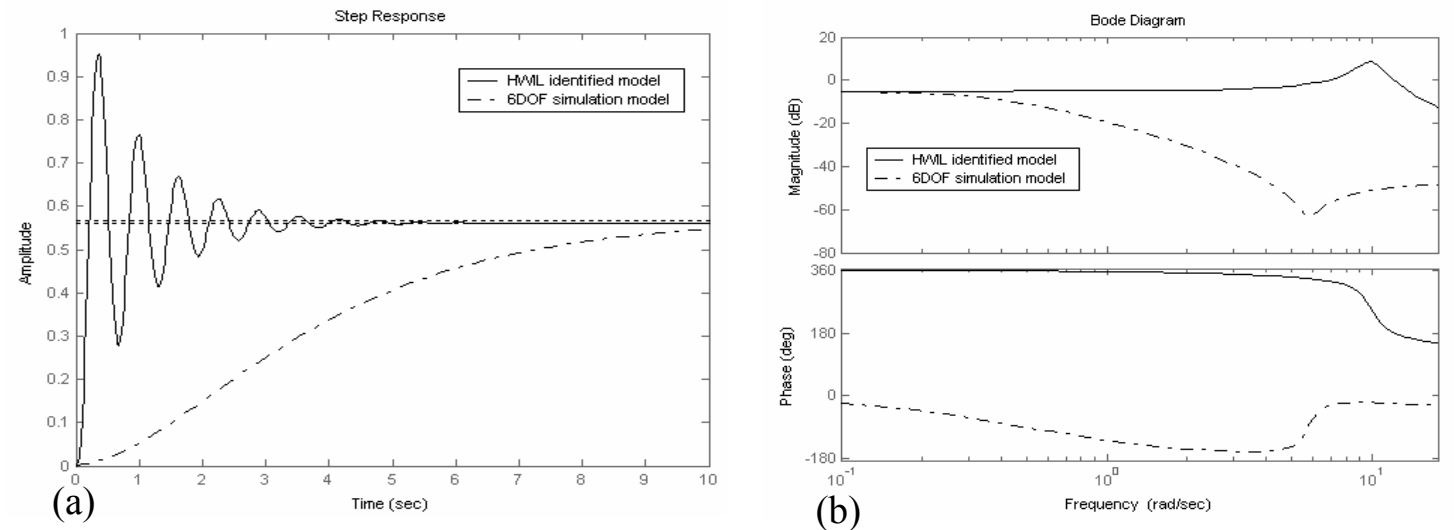

Fig. 11: Control system response at running up phase (a) Step response (b) Bode diagram
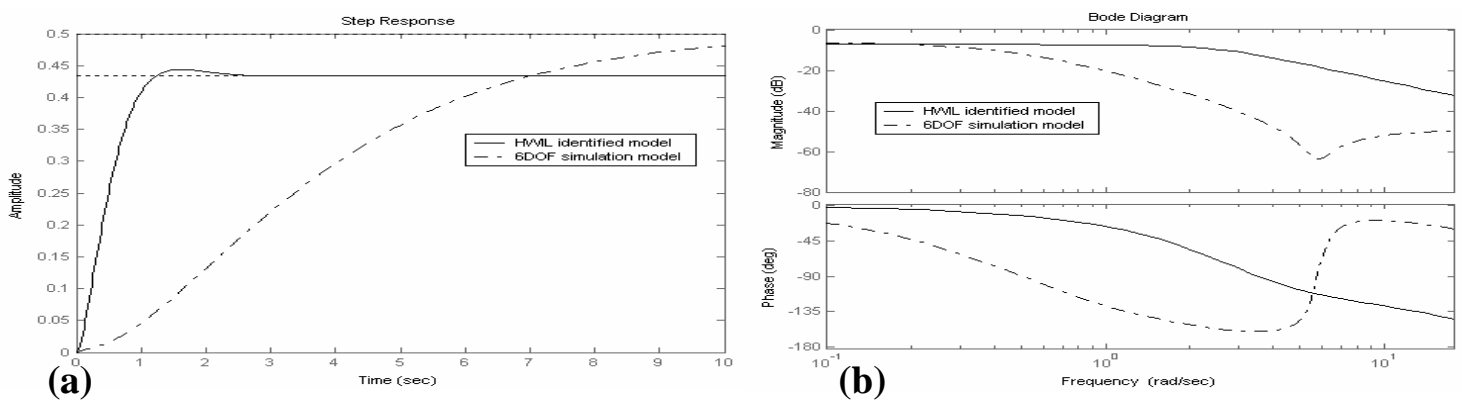

Fig. 12: Control system response at gathering phase (a) Step response (b) Bode diagram
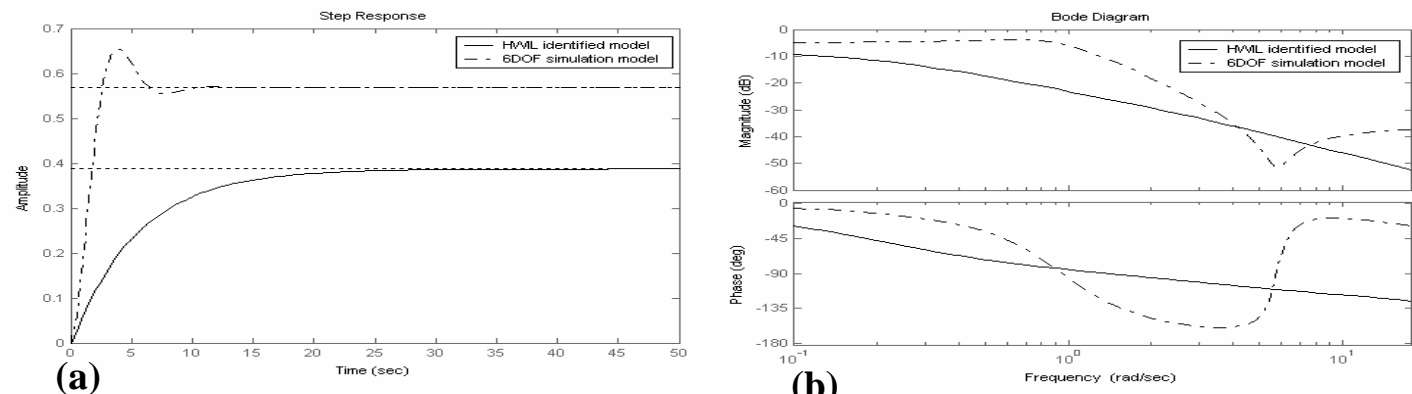

Fig. 13: Control system response at guidance phase (a) Step response (b) Bode diagram 


\section{4 The Missile Guidance Loop}

The result in the previous subsection clarify that the response of the over all guidance loop is sensitive to the variation of the missile inner loop (auto-pilot) response. So it is necessary to find a model for the inner loop much close to the reference. In order to justify the HWIL identified model, a comparison between 6DOF simulation model, HWIL result, identified model and reference trajectories is shown in Fig. 14 for target at range $\left(R_{t x}\right)=2800[\mathrm{~m}]$, velocity $\left(V_{t}\right)=0[\mathrm{~m} / \mathrm{sec}]$, pitch LOS angel $\left(\Psi_{s}\right)=0[\mathrm{mils}]$, and yaw LOS angel $\left(\theta_{s}\right)=0.25$ [mils].

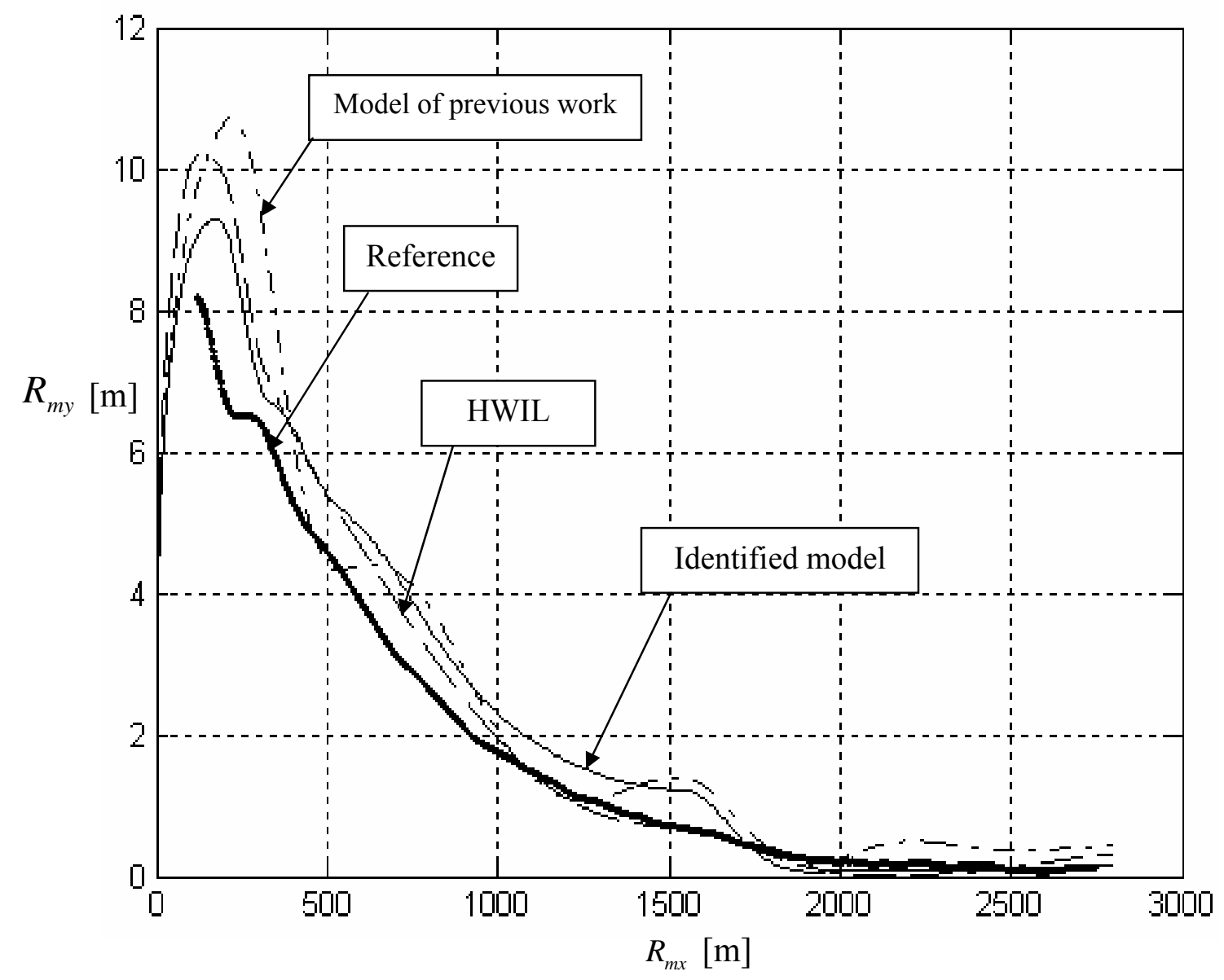

Fig. 14: Flight pitch trajectory of reference, previous simulation model, HWIL, identified model at same flight parameters

This figure shows that the identified model based on hardware in the loop simulation is closer to the reference trajectory with less oscillation than the previous work. This enhancement in the system performance is the first objective of the paper via good and precise modelling.

\section{4- Conclusions}

This paper was devoted to obtain good modeling for the underlying missile system toward enhanced performance. To achieve this objective, the HWIL experiment is described complemented with system identification theory and algorithms. Then, the system 
performance is evaluated using different types of models and at different flight phases. The results showed how the identification model is robust such that the system performance is closer to the reference trajectory with less oscillation than the previous work. In addition, the lesser excursions in the flight path lead to less flight time, possible increased range and less possibility to ground hit.

\section{References}

[1] Astrom K.J. and B. Wittenmark, Adaptive Control, Addison Wesley, 1989.

[2] EL-Sheikh, G.A., Guidance and Control: Theory and Applications, to be Published.

[3] EL-Sheikh, G.A., M.J.Grimble, and M.A.Johanson, on the performance of GH $\infty$ self-Tuning for Aero Engine Control, control-97, warwick University, March 01-07,1997, pp1306-1310.

[4] El-Sheikh, G.A., Recursive Identification Methods, $7^{\text {th }}$ ASAT, M.T.C., Cairo, Egypt, May 13-15, 1997.

[5] Garnell P. and D.J.East, Guided Weapon Control System, Pergamon press 1977.

[6] Greene, M.E. and T.S. Denney, Real-Time Estimator for Control of an Orbiting Single Tether System, IEEE Transactions on Aerospace and Electronic Systems, Vol. 27, No. 6, November 1991.

[7] Gustavsson, L. Ljung and T. Soderstrom, Survey Paper: Identification of Processes in Closed Loop Identifiability and Accuracy Aspects, Automatica, Vol. 13, pp 59-75, 1977.

[8] Hashad, A.I., An Enhancement System For Guidance and Control of IR Command Guided Missiles, M.Sc.thesis, Military Technical College, 1988.

[9] Lawrence, A., Modern Inertial Technology; Navigation, Guidance and Control, Springer Verlag, 1993.

[10]Locke, A.S., Guidance, D.Van Nostrand Company, 1955.

[11]Ljung, L., System Identification: Theory for the User, Prentice-Hall, 1987.

[12]Ljung, L. and T. Soderstrom, Theory and Practice of Recursive Identification, The MIT Press, 1983.

[13]Maintenance, repair and technical documents of the underlying system.

[14]Puckett, A.E., and S.Ramo, Guided Missile Engineering, McGraw Hill, 1959.

[15]Seborg, D.E., T.F. Edgar and S.L. Shah, Adaptive Control Strategies for Process Control: A survey, AICHE Journal, Vol.32, No.6, pp 881-913, June 1986.

[16]Soderstrom and P. Stoica, System Identification, Prentice Hall, 1989.

[17]Weinfeld, R., Towards More Precise Recursive Prediction Error Algorithm, Automatica, Vol. 26, No. 5, pp 925-928, 1990.

[18]Zarchan, P., Tactical and Strategic Missile Guidance, Second Edition, AIAA, 1997.

[19]Zhang, H.G., J.Chen, and H.Y.Zhang, A Recursive Identification Algorithm for Estimating Abrubt Parameter Changes, IEE International Conference Control 1991, number 332, Vol.1 